\title{
Beneficios de la hipoterapia para personas con Trastorno por Déficit de Atención e Hiperactividad en edad escolar. Revisión sistemática exploratoria Benefits of hypotherapy for people with Attention Deficit and Hyperactivity Disorder in school age. Exploratory systematic review
}

\author{
Luisa Gámez-Calvo, José M. Gamonales, Víctor Hernández-Beltrán, Jesús Muñoz-Jiménez \\ Universidad de Extremadura (España)
}

\begin{abstract}
Resumen. El objetivo del presente documento de investigación fue realizar una revisión sistemática exploratoria estructurada basada en los resultados encontrados en torno al término Hipoterapia y Trastorno por Déficit de Atención e Hiperactividad. Para la búsqueda de publicaciones científicas, se utilizaron como descriptores las siguientes palabras clave: Hippotherapy y «ADHD». Los términos fueron introducidos en las bases de datos en lengua inglesa, y se utilizaron las bases de datos informatizadas Scopus, Web Of Science, y Pubmed. Además, sólo se consideraron los Artículos de revista cientificas hasta marzo del 2021. Para limitar la búsqueda se introdujeron cuatro criterios de inclusión: i) Seleccionar solamente Artículos de revista científicas, ii) Mencionar al menos alguna de las características de la Hipoterapia para personas con trastorno por déficit de atención e hiperactividad en edad escolar (mínimo 50 palabras), iii) Estar escrito en idioma español, inglés o portugués y, iiii) estar a texto completo o con disponibilidad de resumen. Tras el Procedimiento de registro para los trabajos de investigación y análisis de datos, se seleccionaron once documentos relacionados con la temática que muestran que las terapias ecuestres son una herramienta beneficiosa para las personas con TDAH en edad escolar. Además, principalmente los documentos son Estudios experimentales con metodología cuantitativa $(n=9)$, y Estudio teóricos $(n=2)$. Por último, los beneficios de la Hipoterapia tienen relación directa con la mejora de la calidad de vida de las personas conTDAH en edad escolar.
\end{abstract}

Palabras clave: Hipoterapia, TDAH, adolescente.

\begin{abstract}
This research document aimed to carry out a structured exploratory systematic review based on the results found around the term Hippotherapy and Attention Deficit Hyperactivity Disorder. For the search for scientific publications, the following keywords were used as descriptors: Hippotherapy and «ADHD». The terms were entered in computerized databases Scopus, Web of Science and Pubmed. Also, only Scientific journal articles up to March 2021 were considered.To limit the search, four inclusion criteria were introduced: i) Select the only article from scientific journals, ii) Mention at least some of the characteristics of Hippotherapy for people with attention deficit hyperactivity disorder (minimum 50 words), iii) Be in writing in Spanish, English or Portuguese and, iiii) be in full text or with abstract availability. After the Registration Procedure for the studies and data analysis, eleven documents related to the subject were selected that show equestrian therapies are a beneficial tool for people with ADHD in school age. Also, mainly the documents are Experimental studies with quantitative methodology $(n=9)$, and Theoretical studies $(n=2)$. Finally, the benefits of Hippotherapy are directly related to improving the quality of life of people with ADHD in school age.
\end{abstract}

Keywords: Hippotherapy, ADHA, teen.

\section{Introducción}

La habilitación y rehabilitación son procesos a través de los cuales las personas con diferentes discapacidades tienen la oportunidad de contar y mantener óptimos niveles de las capacidades físicas, sensoriales, intelectuales, psicológicas y sociales (Jiménez, 2017). Existe un amplio abanico de técnicas y procedimientos para la intervención de estas personas, como el método Bobath o Perfetti (López-Roa \& Moreno-Rodríguez, 2015). Las terapias ecuestres son en la actualidad una de las herra-

Fecha recepción: 30-03-21. Fecha de aceptación: 19-06-21

José M. Gamonales

josemartingamonales@gmail.com mientas terapéuticas más practicadas por personas con diferentes tipos de discapacidad (Fierro-Aldana, TulaGarzón \& Vera-Cárdenas, 2013). Por tanto, las Intervenciones Asistidas con caballos (en adelante, IAC), y en concreto la Hipoterapia, son un tipo de terapia practicada en casi todos los países de mundo de forma regularizada (Del Rosario-Montejo, Molina-Rueda, MuñozLasa \& Alguacil-Diego, 2014), como alternativa y complemento a la rehabilitación y la reeducación. Integra una metodología de trabajo especializada (Uribe, Restrepo \& Yajaira, 2012), que ha mostrado ser beneficiosa para la calidad de vida y el estado de salud general de las personas (Arias, Arias \& Moretin, 2008), con beneficios psicológicos, sociales y educativos que favorecen el desempeño de las actividades de la vida diaria 
(Gámez-Calvo, Gamonales, Silva-Ortiz \& MuñozJiménez, 2020; Gámez-Calvo, Silva-Ortiz, Gamonales \& Muñoz-Jiménez, 2019). Por tanto, la práctica físicodeportiva es beneficiosa para las personas con diversidad funcional, siempre que se desarrolle con los profesionales adecuados, e implicando al entorno familiar y social (Silva-Ortiz, Gamonales, Gámez-Calvo \& MuñozJiménez, 2020). De esta manera, la asistencia a la IAC permite la estimulación y potenciación del ámbito psicomotor, auditivo, de lenguaje y sensorial de las personas con diversidad funcional en edad escolar (OrtegaEspinoza, 2021; Rivera-Ramo, 2020).

Para la realización de IAC, es necesario que el caballo sea un animal manso y entrenado, que permita el acercamiento y el contacto con personas que pueden presentan conductas alteradas, movimientos involuntarios o usan algún dispositivo (bastón o silla de ruedas) (Villasís-Keever \& Pineda-Leguízamo, 2017). El caballo actúa como portador, es decir, puede montarse y desplazar a una persona transmitiéndole estímulos sensoriales altamente considerables que proporcionan informaciones cenestésicas y sensorial al sistema nervioso, activando procesos de orden superior como las reacciones de control, enderezamientos corporales, la modulación del tono muscular y postural, la coordinación y el equilibrio (Gámez-Calvo, Silva-Ortiz et al., 2019). Por ello, la elección de un buen caballo como coterapeuta es fundamental en la rehabilitación (LópezRoa \& Moreno-Rodríguez, 2015). Además, las IAC para personas con diversidad funcional han adquirido popularidad como consecuencias de los grandes beneficios que aporta a los usuarios (White, Zippel \& Kumar, 2020). Por tanto, la participación una vez por semana en programas de IAC ayuda a controlar el peso (Lee, Park, \& Kim, 2015), y mejoran la calidad de vida de forma general a los niños con diversidad funcional (Lee, Park \& Kim, 2017).

En la literatura científica, existen evidencias relacionadas con la eficacia de la Hipoterapia en diferentes colectivos de personas con discapacidad, como personas con parálisis cerebral o daño cerebral adquirido (Fernández-Gutiérrez, Apolo-Arenas, Martínez-García \& Caña-Pino, 2014; Mendizábal-Alonso, 2020; Vargas, Patricio, Solís-Cartas, Martínez-Larrarte \& SerranoEspinosa, 2016; Villasís-Keever \& Pineda-Leguízamo, 2017), personas con retraso psicomotor (Fierro-Aldana et al., 2013; López-Roa, 2011), personas con síndrome de Down (De Miguel, De Miguel, Lucena-Antón \& Rubio, 2018; Gámez-Calvo, Silva-Ortiz et al., 2019), personas con esclerosis múltiple (Eizaguirre, Alonso,
López \& Díaz-Videla, 2020; Muñoz-Lasa, López, AtínArratibel, Bravo-Llatas, Pastor-Jimeno \& Máximo-Bocanegra, 2019), personas con autismo (Fierro-Bósquez, Fierro-Vasco \& Aguinaga, 2018; Tabares, Vicente-Castro, Sánchez-Herrera, Alejo \& Cubero, 2014), o en personas mayores (Gámez-Calvo, Gamonales et al., 2020), entre otros. Por ello, la Hipoterapia es un tratamiento multidisciplinar e integral de base neurológica, que utiliza como herramienta principal al caballo y sus cualidades (movimiento, ritmo o calor), las cuales estimulan múltiples áreas psicomotrices y sensoriales (Shurtleff \& Engsberg, 2012; López-Roa, 2011). Sin embargo, los trabajos de investigación relacionados con Hipoterapia y personas con Trastorno por Déficit de Atención e Hiperactividad (en adelante, TDAH), son escasos. Entendiéndose, el TDAH como una alteración del comportamiento que se diagnóstica normalmente durante la etapa infantil, y persiste en la adolescencia y edad adulta (Giraldo \& Chaves, 2014). Las principales características de las personas con TDAH están relacionadas con el rendimiento escolar, el déficit psicomotor, la dificultad en el área de conducta y las relaciones sociales, así como la carencia de motivación, la impulsividad, el trastorno del sueño y la imagen corporal (Gamonales et al., 2018).

La actividad física, integrada en los tratamientos cognitivo-conductuales, se presenta como una terapia alternativa al tratamiento farmacológico (Orjales \& Polaino-Lorente, 2014). Diferentes trabajos de investigación muestran que el ejercicio físico podría producir beneficios a nivel cognitivo, ejecutivo, de coordinación motora, en el rendimiento académico, en el comportamiento y en las habilidades sociales de los niños con TDAH, así como la posibilidad de reducir los síntomas propios de este trastorno (Wigal, Emmerson, Gehricke \& Galassetti, 2013; Ziereis \& Jansen, 2015; Lomas-Rivera \& Clemente-Remón 2017). Por ello, los animales, en este caso los caballos, facilitan un estado de relajación y tranquilidad inmediata, debido, junto a otros factores, a que captan y mantienen la atención de las personas que les rodean (Fine, 2010; Mallon, Ross, Ross \& Klee, 2010). Esta es una de las razones de emplear terapias asistidas con animales como alternativa a las terapias farmacológicas (Oh et al., 2018; Pérez-Gómez et al., 2020). Por tanto, dentro de los tratamientos no farmacológicos, las actividades y terapias asistidas por equipos son fundamentales para tratar a las personas con TDAH, siendo la Hipoterapia una alternativa excelente para evitar la medicación, puesto que mejora su compromiso, las conductas desadaptativas y la socializa- 
ción (Eraud, 2020).

Ante la escasez de documentos de trabajo de investigación (estudio teórico) sobre esta temática, y analizando las distintas bases de datos, se muestra conveniente realizar una nueva revisión sistemática exploratoria que complete el estado del arte en torno a la investigación en Hipoterapia, en relación con las personas con TDAH, que aporten conclusiones sobre los beneficios de las terapias ecuestres para este colectivo. Por tanto, los objetivos del presente trabajo de investigación fueron: a) realizar una revisión sistemática exploratoria relacionada con la Hipoterapia y personas con TDAH, en edad escolar, y b) conocer los beneficios de las IAC, en concreto la Hipoterapia, en dicho colectivo.

\section{Método}

\section{Diseño}

Esta investigación se ubica dentro de los estudios teóricos (Montero \& León, 2007). Para ello, se realizó una búsqueda y recopilación de documentos actuales sobre el tema de interés, y significativos para el lector (Ato, López \& Benavente 2013), con el objetivo de analizar los documentos relacionados con los beneficios de la Hipoterapia en personas con TDAH, en edad escolar.

\section{Estrategia de búsqueda}

La muestra estuvo formada por Artículos de revistas científicas. Del total de 1210 documentos en la primera fase de búsqueda, entre las tres bases de datos, con el primer término seleccionado Hippotherapy, tras incluir el segundo $A D H D$, se rechazaron 1199, y se seleccionaron 11 documentos que se ajustaban a los criterios de inclusión y exclusión establecidos. Además, se tuvo en cuenta las palabras clave incluidas en los diferentes manuscritos seleccionados. Para la búsqueda de documentos, se utilizaron las bases de datos: Scopus, Web Of Science (en adelante, WOS), y Pubmed.

\section{Criterios para la selección de los estudios}

Para la búsqueda y selección de documentos, se emplearon las palabras clave en lengua inglesa: Hippotherapy y ADHD. Los documentos seleccionados para formar parte de la muestra debían de cumplir una serie de criterios de inclusión (Tabla 1).

\section{Codificación de las variables}

Se clasificaron los trabajos de investigación atendiendo a los siguientes criterios: Título, Autor/es, Año de publicación, Palabras Clave, Base de datos, Resumen, Tipo de estudio, Muestra, Variables de los Artículos de revista científicas seleccionados, Protocolo de intervención, Beneficios de la Hipoterapia y Calidad de los documentos seleccionados (Tabla 2).

\section{Procedimiento de registro para los estudios}

El procedimiento utilizado en este trabajo de investigación es similar a los existentes en la literatura científica para personas con diferentes capacidades (GámezCalvo, Gamonales et al., 2020; Gamonales, Durán-Vaca, Gámez-Calvo, Hernández-Beltrán \& Muñoz-Jiménez, 2021; Gamonales, Muñoz-Jiménez, León \& Ibáñez, 2018). Por ello, una adecuada planificación de la búsqueda literaria favorece su éxito (Thomas, Silverman \& Nelson, 2015), y permite extraer conclusiones relevantes (Gamonales, Gil-Sánchez et al., 2018; Silva-Ortiz

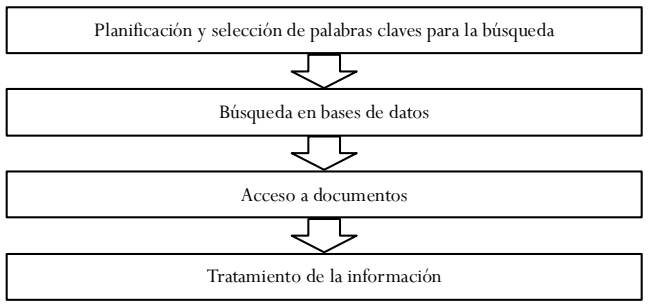

Figura 1. Esquema representativo del proceso de búsqueda de la literatura en Hipoterapia yTDAH 


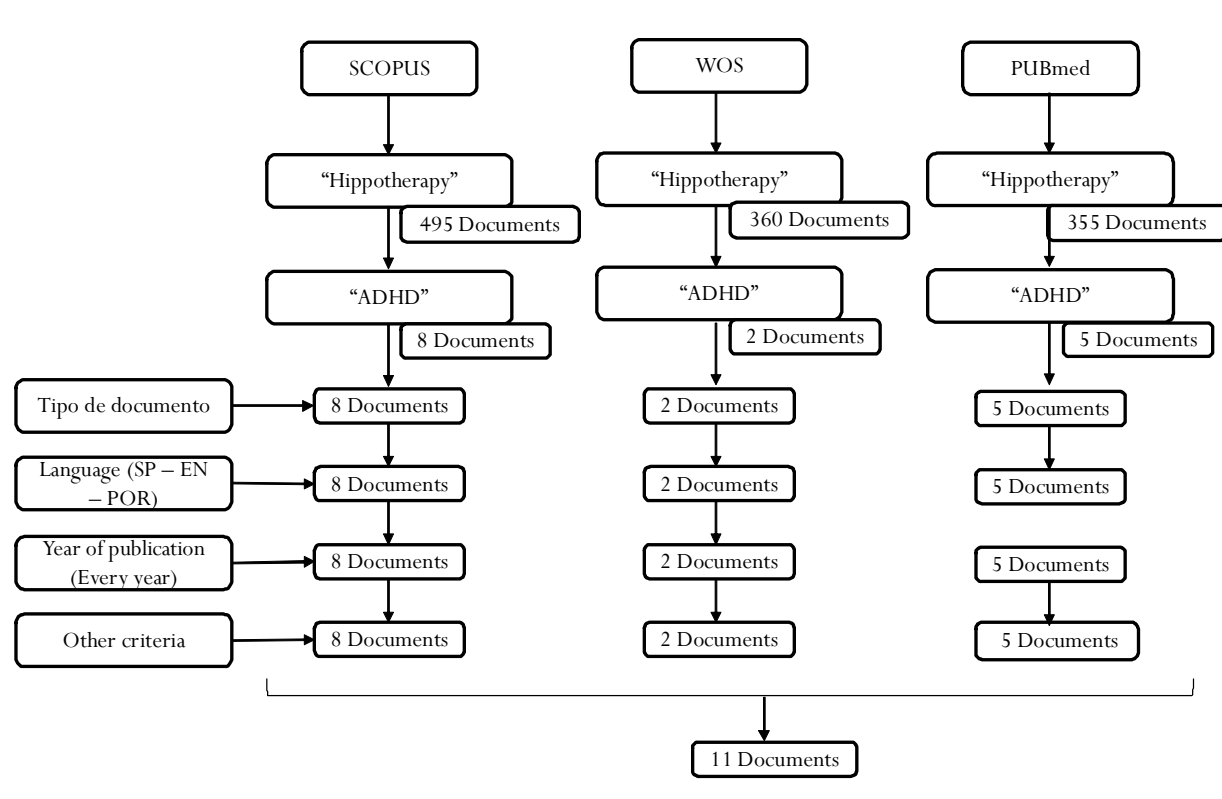

Figura 2. Esquema de documentos seleccionados relacionados con la Hipoterapia y TDAH en las distintas bases de datos. para conocer su vinculación con la temática del trabajo de investigación (Gamonales, MuñozJiménez et al., 2018; Gamonales et al., 2021), así como un análisis de los documentos en función del Año de publicación. En la Figura 3, se muestran las principales Palabras clave utilizadas en los Artículos de revistas científicas. Por último, los manuscritos seleccionados fueron analizados por dos expertos en la temática (Doctores en Ciencias del Deporte y amplia experiencia en personas con discapacidad), con la finalidad de conocer la calidad metodológica y evitar el riesgo de sesgos de estos (Law et al., 1998). Además, fueron seleccionados de forma deliberada e intencional. Para ello, se empleo un cuestionario validado y compuesto por 16 preguntas de respuesta Sí/No relacionadas con los antecedentes, el diseño, la muestra, los resultados y los métodos empleados en los documentos seleccionados. Además, permite clasificar a los Artículos de revistas científicas como:A) Excelente calidad metodológica (Puntuación > 75\%), B) Buena calidad metodológica (Puntuación entre 51\% y 75\%), y C) Baja calidad metodológica (Puntuación <50\%) (Sarmento et al., 2018).

\section{Análisis estadístico}

Se realizó un análisis descriptivo relacionado con las variables Palabras claves y Año de la publicación, con el objetivo de obtener información relevante. Para el análisis estadístico, se utilizó el software Statistical Package of Social Science (versión 24, 2016; IBM Corp., IBM SPSS Statistics para MAC OS, Armonk, NY, EEUU).

\section{Resultados}

Los resultados se muestran en el mismo orden en el que se desarrolló la revisión sistemática exploratoria. En la Tabla 3, aparecen los distintos Artículos de revistas científicas seleccionados hasta marzo del 2021. Además, se muestran las principales características entorno a los beneficios de la Hipoterapia en personas con TDAH, siguiendo los criterios definidos en el método.

En la Figura 4, se muestra el número de documen- 
Tabla 3.

Principales documentos relacionados con los beneficios de la Hipoterapia en personas conTDAH en edad escolens.

\begin{tabular}{|c|c|c|c|c|c|c|c|c|c|}
\hline Título & $\begin{array}{c}\text { Autor } / e \\
s\end{array}$ & Año & $B D$ & Resumen & $T E$ & Muestra & Variables & P.I. & Beneficios \\
\hline $\begin{array}{l}\text { The effect of equine-assisted } \\
\text { therapies on behavioural, } \\
\text { psychological and physical } \\
\text { symptoms for children with } \\
\text { attention deficit/hyperactivity } \\
\text { disorder: A systematic review. }\end{array}$ & $\begin{array}{l}\text { White et } \\
\text { al. }\end{array}$ & $\begin{array}{l}\text { 2020ADHA; animal- } \\
\text { assisted therapy; } \\
\text { attention } \\
\text { déficit/hyperactivity } \\
\text { disorder; Equine- } \\
\text { assisted theraoy; } \\
\text { Hippotherapy }\end{array}$ & $\begin{array}{l}\text { Scopus/ } \\
\text { WOS/ } \\
\text { PubMed }\end{array}$ & $\begin{array}{l}\text { Revisión sistemática sobre la } \\
\text { efectividad de las TAC como } \\
\text { tratamiento para los síntomas } \\
\text { comportamentales, psicológicos y } \\
\text { físicos en niños con TDAH. }\end{array}$ & & $\begin{array}{l}10 \text { documentos } \\
\text { relacionados con } \\
\text { actividades y } \\
\text { terapias asistidas } \\
\text { por equipo en } \\
\text { niños con TDAH }\end{array}$ & $\begin{array}{l}\text { Estudio, Año, Tamaño de la } \\
\text { muestra, Edad, Diagnóstico } \\
\text { Medidas, Protocolo de } \\
\text { intervención (Frecuencia, } \\
\text { Duración e Intensidad), y } \\
\text { Calidad de los documentos } \\
\text { seleccionados. }\end{array}$ & $\begin{array}{l}\text { Intervenciones de } \\
\text {,TAC entre } 40 \text { min y } \\
90 \text { minutos durante } \\
12 \text { semanas. Sesiones } \\
\text { entre } 1 \text { y } 2 \text { semanales }\end{array}$ & $\begin{array}{l}\text { Efectos beneficiosos sobre los } \\
\text { principales sintomas del } \\
\text { TDAH. Mejora en la calidad } \\
\text { de vida y disfrute en general. }\end{array}$ \\
\hline $\begin{array}{l}\text { quine-assisted activities and } 1 \\
\text { herapies in children with } \\
\text { ttention-deficit/hyperactivity } \\
\text { lisorder: A systematic review. }\end{array}$ & $\begin{array}{l}\text { Pérez- } \\
\text { Gómez } \\
\text { yet al. }\end{array}$ & $\begin{array}{l}\text { 2020Animal-assisted } \\
\text { therapy; equine- } \\
\text { assisted therapy; } \\
\text { hippotherapy; } \\
\text { horseback riding } \\
\text { therapy; personal } \\
\text { relationships; quality } \\
\text { of life }\end{array}$ & PubMed & $\begin{array}{l}\text { Revisión sistemática para } \\
\text { actualizar el conocimiento sobre } \\
\text { las TAC en niños con TDAH }\end{array}$ & Est. T. & $\begin{array}{l}9 \text { documentos } \\
\text { relacionados con } \\
\text { actividades y } \\
\text { terapias asistidas } \\
\text { por equipo en } \\
\text { niños con } \\
\text { TDAH. } \\
\end{array}$ & $\begin{array}{l}\text { Estudio, Año, Muestra, } \\
\text { Edad, Área geográfica, } \\
\text { Tratamiento de control, } \\
\text { Tratamiento experimental, } \\
\text { Tiempo de IAC, Duración, } \\
\text { Número de sesiones por } \\
\text { semana, Sesiones totales y } \\
\text { Calidad de los documentos } \\
\text { seleccionados. }\end{array}$ & $\begin{array}{l}\text { Intervenciones de } \\
\text { TAC entre } 15 \text { min y } \\
40 \text { minutos durante } 4 \\
\text { semanas. Sesiones } \\
\text { entre } 1 \text { y } 3 \text { semanales }\end{array}$ & $\begin{array}{l}\text { No hay suficientes estudios } \\
\text { con buena calidad } \\
\text { metodológica para } \\
\text { determinar si las TAC son una } \\
\text { intervención eficaz para el } \\
\text { tratamiento del TDAH }\end{array}$ \\
\hline $\begin{array}{l}\text { Self-Management Intervention } \\
\text { for Attention and Executive } \\
\text { Functions Using Equine- } \\
\text { Assisted Occupational } \\
\text { Therapy among Children } \\
\text { Aged 6-14 Diagnosed with } \\
\text { Attention }\end{array}$ & $\begin{array}{l}\text { nGilboa \& } \\
\text { Helmer }\end{array}$ & $\begin{array}{l}\text { 2020ADHA; cognitive } \\
\text { approach; equine- } \\
\text { assisted activities and } \\
\text { therapies; executive } \\
\text { functions; functional } \\
\text { goals; ocupational } \\
\text { therapy }\end{array}$ & Scopus & $\begin{array}{l}\text { Examina la efectividad de la } \\
\text { terapia ocupacional a través de las } \\
\text { TAC en el autocuidado y las } \\
\text { funciones ejecutivas de los niños } \\
\text { con TDAH. }\end{array}$ & $\begin{array}{l}\text { Emp. } \\
\text { s Cuant. }\end{array}$ & $\begin{array}{l}25 \text { niños con } \\
\text { TDAH. Entre } 6 \text { y } \\
14 \text { años. }\end{array}$ & $\begin{array}{l}\text { Características } \\
\text { ydemográficas, } \\
\text { Comportamiento, Función } \\
\text { ejecutiva (BRIEF), y } \\
\text { Desempeño Ocupacional } \\
\text { (COPM) }\end{array}$ & $\begin{array}{l}\text { Intervenciones de } \\
\text { TAC de } 45 \text { min } \\
\text { durante } 12 \text { semanas. } 1 \\
\text { sesión semanal. }\end{array}$ & $\begin{array}{l}\text { Mejora en las funciones } \\
\text { ejecutivas, el desempeño } \\
1 \text { ocupacional y la mejora de la } \\
\text { satisfacción. Junto con la } \\
\text { mejora de las metas } \\
\text { personales. }\end{array}$ \\
\hline
\end{tabular}

Attention

therapy

\begin{tabular}{l} 
Disorder. \\
\hline Efficacy of Hippotherapy Oh et al. 2018Animal-assited $\quad$ Scopus/ Investigar los efectos de la $\quad$ Emp. 34 niños con $\quad$ Síntomas deTDAH, Entrenamiento de Mejora de los síntomas de C
\end{tabular}

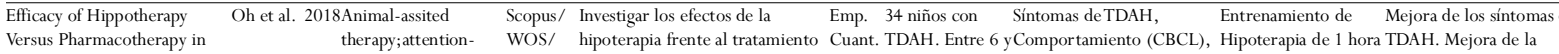

Attention- deficit/hyperactivity PubMed farmacológico para niños con 12 años. Autoestima, de duración 2 atención, de la impulsividad

Deficit/Hyperactivity $\quad$ disorder; TDAH. Calidad de vida, $\quad$ veces/semana, de la calidad de vida.

Disorder: A Randomized $\quad$ hippotherapy; $\quad$ Coordinación y Función durante 12 semanas.

$\begin{array}{llll}\text { Clinical Trial. } & \text { psychopharmacothera } & \text { cerebral. } & \text { Tratamiento }\end{array}$

py farmacológico en el

\begin{tabular}{llllll} 
& & & grupo control. & \\
\hline Hippotherapy and & Lee et 2017Hippotherapy; brain PubMed Investigar los efectos de la & Emp. 16 niños con & Nivel de BDNF en sangre, Entrenamiento & El entrenamiento combinado C
\end{tabular}

neurofeedback training effect al. function; ADHD Hipoterapia y el neurofeedback Cuant. TDAH. 8 en el Función cerebral, EEG, y primario: 1 o 2 de Hipoterapia y

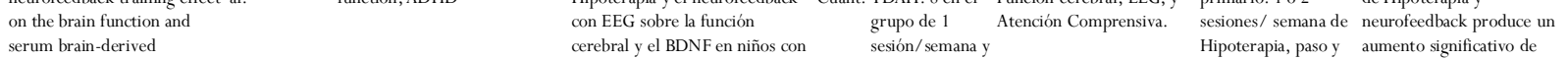

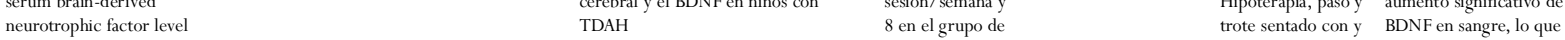

$\begin{array}{lll}\text { changes in children with } & 2 \text { sesiones } / & \text { sin estribos. }\end{array}$

$\begin{array}{lll}\text { changes in children with } & 2 \text { sesiones/ } & \text { puede inducir a una mejor } \\ \text { attention-deficit or/and } & \text { Semana. } & \text { Entribos. } \\ \text { de la función cerebral en }\end{array}$

$\begin{array}{ll}\text { hyperactivity disorder } & \text { secundario: } 1 \text { o } 2 \text { niños con TDAH. }\end{array}$

sesiones/semana de

Hipoterapia y

entrenamiento de
neurofeedback

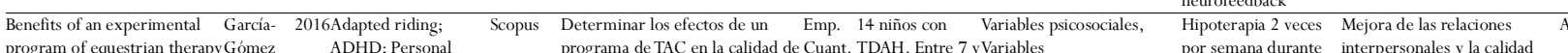

program of equestrian therapy Gómez ADHD; Personal programa de TAC en la calidad de Cuant. TDAH. Entre 7 yVariables por semana durante interpersonales y la calida

for children with ADHD. et al. relationship; Quality vida y variables psicosociales de 14 años. comportamentales, Calidad 12 semanas, sesiones de vida.

of life niños con TDAH.

The effects of equine-assisted Yoo et $\quad$ 2016Attention deficit $\quad$ Scopus $\quad$ Investigar los efectos de las TAC Emp. 22 niños con $\quad$ Síntomas deTDAH, TAC 2 veces por $\quad$ Mejora de los síntomas de A

\begin{tabular}{|c|c|c|c|c|c|c|c|}
\hline $\begin{array}{l}\text { The effects of equine-assisted Yoo et } \\
\text { activities and therapy on al. } \\
\text { resting-state brain function in } \\
\text { attention-deficit/hyperactivity } \\
\text { disorder: A pilot study. }\end{array}$ & $\begin{array}{l}\text { 2016Attention deficit } \\
\text { hyperactivity } \\
\text { disorder; Default } \\
\text { mode network; } \\
\text { Equine-assisted } \\
\text { therapy; Functional } \\
\text { magnetic resonance }\end{array}$ & Scopus & $\begin{array}{l}\text { Investigar los efectos de las TAC } \\
\text { en participantes con TDAH, } \\
\text { comparando la resonancia } \\
\text { magnética funcional e estado de } \\
\text { reposo y sus correlaciones } \\
\text { clínicas. }\end{array}$ & $\begin{array}{l}\text { Emp. } 22 \text { niños con } \\
\text { Cuant. TDAH (solo } 10 \\
\quad \text { finalizaron la } \\
\text { investigación). }\end{array}$ & $\begin{array}{l}\text { Síntomas deTDAH, } \\
\text { Función cerebral, y } \\
\text { Conectividad neuronal. }\end{array}$ & $\begin{array}{l}\text { TAC } 2 \text { veces por } \\
\text { semana durante } 12 \\
\text { semanas con una } \\
\text { duración de } 1 \\
\text { hora/sesión. 20-30 } \\
\text { min de monta. }\end{array}$ & $\begin{array}{l}\text { Mejora de los sintomas de } \\
\text { TDAH. Mejora de la } \\
\text { conectividad neuronal } \\
\text { funcional de áreas } \\
\text { determinadas del cerebro. }\end{array}$ \\
\hline
\end{tabular}

imaging; Regional

homogeneity; Resting

state

\begin{tabular}{|c|c|c|c|c|c|c|c|c|c|}
\hline $\begin{array}{l}\text { Changes in Gait Balance and } \\
\text { Brain Connectivity in } \\
\text { Response to Equine-Assisted } \\
\text { Activity and Training in } \\
\text { Children with Attention } \\
\text { Deficit Hyperactivity } \\
\text { Disorder. } \\
\end{array}$ & $\begin{array}{l}\text { Hyun et } \\
\text { al. }\end{array}$ & $\begin{array}{l}\text { 2016Gait Balance; Bran } \\
\text { Connectivity; Equine- } \\
\text { Assisted Therapy; } \\
\text { Attention-Deficit } \\
\text { Hyperactivity } \\
\text { Disorder; ADHD }\end{array}$ & Scopus & $\begin{array}{l}\text { Investigar como las TAC pueden } \\
\text { mejorar los síntomas clínicos y el } \\
\text { equilibrio de la marcha en niños } \\
\text { con TDAH, y si estas mejoras } \\
\text { están asociadas a un aumento de } \\
\text { la conectividad cerebral en el } \\
\text { circuito del equilibrio. }\end{array}$ & $\begin{array}{l}\text { Emp. } \\
\text { Cuant. }\end{array}$ & $\begin{array}{l}12 \text { niños con } \\
\text { TDAH de } 12 \\
\text { años. }\end{array}$ & $\begin{array}{l}\text { Síntomas de TDAH, Presión } \\
\text { plantar, Conectividad } \\
\text { cerebral, } \\
\text { Equilibrio de la marcha, y } \\
\text { Análisis demográfico. }\end{array}$ & $\begin{array}{l}\text { TAC } 3 \text { veces por } \\
\text { semana durante } 4 \\
\text { semanas, con una } \\
\text { duración de } 70 \\
\text { minutos. } 40 \text { min de } \\
\text { monta. }\end{array}$ & $\begin{array}{l}\text { Mejora de los clínicos, el } \\
\text { equilibrio de la marcha y la } \\
\text { conectividad cerebral que } \\
\text { controla el equilibrio de la } \\
\text { marcha. }\end{array}$ \\
\hline $\begin{array}{l}\text { Effects of hippotherapy on } \\
\text { brain function, BDNF level, } \\
\text { and physical fitness in childrer } \\
\text { with ADHD }\end{array}$ & $\begin{array}{l}\text { Lee, } \\
\text { Park \& } \\
\text { Kim }\end{array}$ & $\begin{array}{l}\text { 2015Hippotherapy, } \\
\text { ADHD, brain-derived } \\
\text { neurotropic factor }\end{array}$ & PubMed & $\begin{array}{l}\text { Investigar los efectos de la } \\
\text { Hipoterapia en la función } \\
\text { cerebral y BDNF en sangre en } \\
\text { niños con TDAH }\end{array}$ & $\begin{array}{l}\text { Emp. } \\
\text { Cuant. }\end{array}$ & $\begin{array}{l}39 \text { niños con } \\
\text { TDAH. } 20 \text { en el } \\
\text { grupo de TAC y } \\
19 \text { en el grupo }\end{array}$ & $\begin{array}{l}\text { Aptitud Física, Actividad } \\
\text { cerebral y BDNF }\end{array}$ & $\begin{array}{l}\text { Programa de } \\
\text { Hipoterapia } \\
\text { combinada con } \\
\text { equitación }\end{array}$ & $\begin{array}{l}\text { No muestra beneficios } \\
\text { significativos. Sugiere que el } \\
\text { entrenamiento de } \\
\text { Hipoterapia debe ser }\end{array}$ \\
\hline
\end{tabular}

$\begin{array}{lll}\text { with ADHD } & \text { cerebral y BDNF en sangre en } & \text { grupo de TAC y } \\ & \text { niños con TDAH } & 19 \text { en el grupo }\end{array}$

terapion ser

$\begin{array}{ll}\text { terapeutica. } 1 \text { sesion } & \text { modificado y desarrollado } \\ \text { semanal durante } 32 & \text { para aumentar su eficacia en }\end{array}$

semanas, con una niños con TDAH.

duración de 1 hora

(15 min

calentamiento, 35

min principal y 10

min vuelta a la

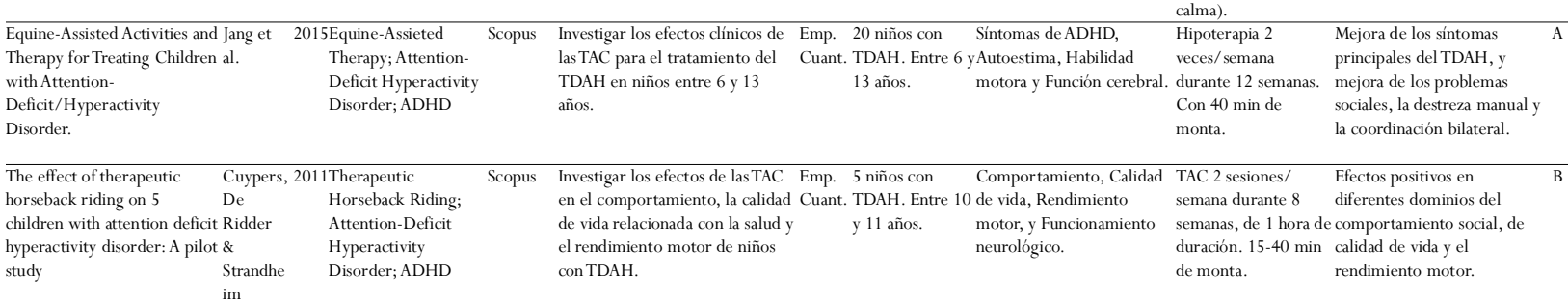

$\frac{\mathrm{im}}{\text { BD: Base de datos, TE: Tipo de estudio; Emp. Cuant.: Estudio Experimental con metodología Cuantitativa; Est. T.: Estudio Teórico; TAC: Terapias Asistidas con Caballos; TDAH: Trastorno por Déficit de Atención e }}$ Hiperactividad. CD: Calidad de los documentos seleccionados; A: Excelente calidad metodológica; B: Buena calidad metodológica; C: Baja calidad metodológica; EEG: Electroencefalografía; P.I.: Protocolo de intervención. 
tos relacionados con Hipoterapia y personas con TDAH en función del Año de la publicación. Como se puede apreciar, es en el año 2016 y 2020, donde existen el mayor número de publicaciones relacionadas con la temática objeto del trabajo de investigación. Además, durante el intervalo del 2012 al 2014 no existen publicaciones, así como en el año 2019.

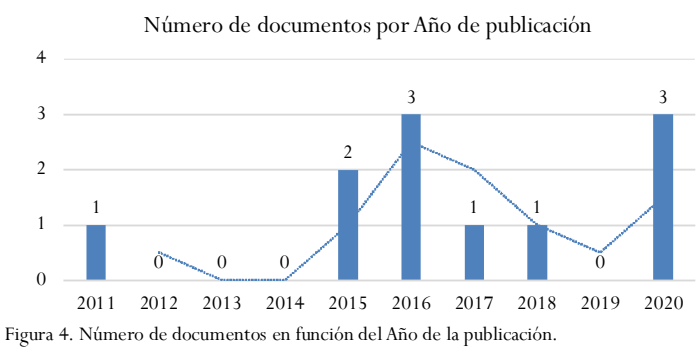

\section{Discusión}

El objetivo del presente trabajo de investigación fue realizar una revisión sistemática exploratoria relacionada con la Hipoterapia y las personas con TDAH, con el fin de analizar el estado del conocimiento sobre los beneficios de la Hipoterapia, identificando y clasificando la información más relevante. Sirve de base para futuras revisiones, al tiempo que permite extraer conclusiones. Los documentos seleccionados muestran una gran variedad de métodos de investigación en los diferentes trabajos de investigación, desde Estudios teóricos hasta Estudios experimentales con metodología cuantitativa. Todos ellos, coinciden en que la Hipoterapia es un tratamiento beneficioso para la mejora de los principales síntomas del TDAH. Para la selección de los 11 documentos finales que cumplían con los criterios de inclusión y exclusión previamente propuestos por los investigadores, así como en el procedimiento utilizado en la revisión de los manuscritos, se ha seguido procesos similares a los existentes en la literatura (Gámez-Calvo et al., 2020; Gamonales, Gil-Sánchez et al., 2018; Gamonales, Muñoz-Jiménez et al., 2018; Gamonales et al., 2021), con la finalidad de seleccionar los Artículos de revistas científicas más adecuados a la temática objeto del trabajo de investigación.

Los resultados relacionados con el Año de la publicación muestran que el año 2016 es el periodo donde se encuentran el mayor número de publicaciones. En la literatura científica, no existe documentos similares donde se muestren los principales Artículos de revistas científicas en función de las variables aplicadas en este trabajo de investigación. Por ello, se recomienda ampliar el campo de conocimiento objeto del manuscrito de in- vestigación, e innovar en los procedimientos de búsqueda de documentos. Es fundamental seguir una estructura de investigación similar a las existentes, como la que se ha desarrollado Gámez-Calvo et al., (2020), o Gamonales, Gil-Sánchez et al., (2018), con la finalidad de realizar Estudios Teóricos de forma óptima de una manera narrativa, o bien plantearse de manera sistemática. Además, es clave dar un tratamiento personalizado a los manuscritos de revisión. Por tanto, sería recomendable diseñar y validar nuevos procesos de revisión específicos para el ámbito de las Ciencias del Deporte, y evitar la utilización de algunas metodologías existentes como el método PRISMA, con un conjunto mínimo de elementos, basados en evidencias para crear documentos de revisiones sistemáticas y meta-análisis aplicados para cualquier campo de conocimiento. Además, otra de la limitación metodológica del modelo PRISMA es el sesgo de publicación. Es decir, muchos trabajos de investigación que obtienen resultados negativos o tardan en publicarse son más difíciles de identificar (PérezRodríguez, 2012).

Respecto a los resultados relacionados con las Palabras clave, se puede apreciar que los principales vocablos son: ADHD $(n=8)$, Attention Déficit Hyperactivity Disorder $(n=6)$, Hippotherapy $(n=6)$, y Equine-Assisted Therapy $(n=5)$. En la literatura cientifica, no existen documentos que corroboren los resultados obtenidos. Sin embargo, los términos extraídos están vinculados con la temática objeto de investigación. Este análisis estadístico puede servir para determinar las Palabras clave del documento de revisión realizado, como sugieren los autores Gamonales, Muñoz-Jiménez et al., (2018), en su investigación vinculada con la literatura en $\mathrm{Fa} 5$, donde las principales Palabras clave están relacionadas con su temática de investigación. Por tanto, este análisis estadístico se muestra óptimo para conocer si los documentos seleccionados están en consonancia con el/los objetivo/s del trabajo propuesto.

En relación con las Bases de datos empleadas, los resultados muestran que la mayor parte de los Artículos de revistas científicas seleccionados están en la plataforma Scopus $(n=8)$, y en menor número en el resto de las bases de datos, $\operatorname{WOS}(n=2)$, y Pubmed $(n=5)$. Es decir, existen documentos que se encuentran en varias bases de datos. Estos resultados pueden ser consecuencia de seleccionar una base de datos bibliográficas de resúmenes y citas de Artículos de revistas científicas, así como por la elección del criterio de inclusión de documentos: «Seleccionar Artículo de revistas científicas». En la literatura científica, existen Estudios teóricos que emplean procesos 
de inclusión y exclusión para seleccionar manuscritos similares (Gámez-Calvo, Gamonales et al., 2020; Gamonales, Gil-Sánchez et al., 2018; Gamonales, Muñoz-Jiménez et al., 2018). Sin embargo, la mayoría de los documentos utilizan criterios de inclusión generales como "Seleccionar cualquier tipo de documento científico». Por ello, se recomienda diseñar criterios de selección de documentos específicos en función de la temática, y según lo/s objetivo/s del estudio, con la finalidad de seleccionar los manuscritos más adecuados con la investigación.

Los resultados relacionados con el Tipo de estudio muestran que la mayoría de los documentos seleccionados son Estudio experimental con Metodología cuantitativa $(n=9)$, y Estudio teórico $(n=2)$. Los estudios experimentales incluidos en el estudio de revisión sistemática exploratoria relacionados con la Hipoterapia y las personas con TDAH en edad escolar muestran una reducida muestra, siendo el documento de Oh et al., (2018), el que presenta un mayor número de sujetos $(n=34)$, y el de menor muestra, el llevado a cabo por Cuypers et al., (2011), contando sólo con 5 participantes. Otro aspecto para destacar de los estudios experimentales es el empleo de diferentes instrumentos para la recogida de datos, desde cuestionarios y escalas clínicas (GarcíaGómez et al., 2016; Gilboa \& Helmer, 2020; Cuypers et al., 2011), hasta escáneres cerebrales, resonancia magnética o electroencefalografía (Hyun et al., 2016; Oh et al., 2018; Yoo et al., 2016). La duración de la intervención y de las sesiones realizadas en los estudios experimentales, son mayoritariamente de una o dos sesiones semanales de aproximadamente 40-60 minutos. Y, siendo, el programa con mayor duración de 32 semanas (Lee et al., 2015). Sin embargo, en los Estudios teóricos varían la duración de los protocolos de intervención en función de los autores de los documentos (Pérez Gómez et al., 2020; White et al., 2020). Por tanto, la principal limitación de los estudios experimentales es su reducida muestra, así como la no estandarización de la duración de las sesiones, lo que no permite generalizar los resultados. Los autores de dichas investigaciones utilizan una amplia gama de herramientas de investigación, con la finalidad de recopilar, resumir o anotar los contenidos de las fuentes o datos utilizados para generar conocimiento científico. Por ello, sería interesante ampliar el conocimiento relacionado con el objeto de investigación (ampliar el número de manuscritos), así como conocer en profundidad los hábitos diarios de los sujetos, puesto que pueden afectar a los resultados de las investigaciones. Además, se necesitan más estudios para aclarar si las IAC son eficaces para las personas con TDAH, en edad escolar (Pérez Gómez et al., 2020).

Respecto a los resultados relacionados con los Beneficios de la Hipoterapia muestran que las personas con TDAH, en edad escolar, que asisten a las IAC cuentan con una alternativa eficaz a los tratamientos farmacológicos (Orjales \& Polaino-Lorente, 2014), con efectos positivos a nivel físico, psicológico y social, incluso reduciendo los síntomas propios del trastorno (Wigal et al., 2013; Ziereis \& Jansen, 2015; LomasRivera \& Clemente-Remón 2017). Por tanto, los programas de Hipoterapia para personas con TDAH producen mejoras relacionadas con la calidad de vida y el bienestar en general (Cuypers et al., 2011; GarcíaGómez et al., 2016; Oh et al., 2018; White et al., 2020). Siendo, necesario realizar una progresión: durante las primeras semanas de entrenamiento, deben experimentar y aprender a caminar, trotar y galopar. Posteriormente, deberán realizar ejercicios de postura de medio asiento, y en las últimas semanas del programa, las personas con TDAH, en edad escolar, deben trotar de forma independiente (Lee et al., 2015). Además, la Hipoterapia combinada con diversas intervenciones psicológicas seria útil para mejorar la función cerebral en niños con TDAH (Lee et al., 2017). Sin embargo, es necesario desarrollar nuevas investigaciones en torno a los beneficios de la Hipoterapia y la equitación terapéutica en personas con TDAH, en edad escolar, con muestras más numerosas, un mayor nivel de detalle sobre las intervenciones llevadas a cabo, más duraderas en el tiempo y que contengan evaluaciones cuantificables y contrastables, con el fin de dotar de una mayor evidencia que permita reconocer a la Hipoterapia como tratamiento eficaz como alternativa válida en el tratamiento de niños en edad escolar (Pérez Gómez et al., 2020).

Por último, los resultados relacionados con la Calidad de los documentos seleccionados muestran que la mayoría de los Artículos de revistas científicas tienen una Excelente calidad metodológica $(n=6)$. Además, existen documentos con Buena calidad metodológica $(\mathrm{n}=1)$, y Baja calidad metodológica $(n=3)$. Sin embargo, son escasos como consecuencia de la falta de homogeneidad y baja calidad metodológica, imposibilitando realizar un meta-análisis, ni establecer cuáles son los principales beneficios de las IAC en personas con TDAH (Pérez Gómez et al., 2020). Por ello, se recomienda el análisis de observadores externos al trabajo de investigación, con la finalidad de minimizar los sesgos y, por tanto, obtener resultados fiables de los que extraer conclusiones y tomar decisiones óptimas como hicieron los autores Sarmento et al., 
(2018), en su revisión sistemática sobre las tendencias de investigación en el fútbol. Además, recomendar la selección de jueces expertos de forma deliberada e intencional, puesto que permite aumentar la calidad de la revisión sistemática exploratoria relacionada con los beneficios de la Hipoterapia en personas con TDAH.

\section{Conclusiones}

Tras la revisión sistemática exploratoria, se concluye que la Hipoterapia puede considerarse beneficiosa para las personas con TDAH, en edad escolar, si se realizan de manera adecuada.

La Hipoterapia produce mejoras en los principales síntomas del TDAH, y permite mejorar las funciones ejecutivas, el desempeño social y ocupacional, la atención, la impulsividad, el equilibrio, la coordinación bilateral y la destreza manual. Estos beneficios tienen como consecuencia la mejora de la calidad de vida.

Se recomienda este tipo de terapia asistida con animales con la finalidad de mejorar a nivel biológico y psicosocial. Aún así, los documentos actuales entorno a las IAC son escasos y presentan debilidades metodológicas. Además, los estudios teóricos en torno a las IAC se centran en describir los protocolos de intervención en personas con TDAH, pero ningún manuscrito concluye de forma específica la frecuencia, duración e intensidad de estos. Por ello, se recomienda aumentar los estudios relacionadas con la Hipoterapia y las personas con TDAH, en edad escolar. Pues, la principal limitación de este estudio fue la escasa literatura específica relacionada con la temática.

\section{Agradecimientos y financiación}

Trabajo desarrollado dentro del Grupo de Optimización del Entrenamiento y Rendimiento Deportivo (GOERD) de la Facultad de Ciencias del Deporte de la Universidad de Extremadura. Además, este trabajo ha sido parcialmente subvencionado por la Ayuda a los Grupos de Investigación (GR18170), de la Junta de Extremadura (Consejería de Empleo e Infraestructuras); con la aportación de la Unión Europea a través de los Fondos Europeos de Desarrollo Regional (FEDER).

\section{Referencias}

Arias, V., Arias, B., \& Moretin, R. (2008). Terapia asistida por caballos: nueva propuesta de clasificación, programas para personas con discapacidad intelectual y buenas prácticas. Revista Española sobre Discapacidad In- telectual, 39(2), 18-30.

Ato, M., López, J.J., \& Benavente, A. (2013). Un sistema de clasificación de los diseños de investigación en psicología. Anales de Psicología, 29(3), 1038-1059.

Cuypers, K., De Ridder, K., \& Strandheim, A. (2011). The effect of therapeutic horseback riding on 5 children with attention deficit hyperactivity disorder: A pilot study. Journal of Alternative and Complementary Medicine, 17(10), 901-908. https://doi.org/ 10.1089/acm.2010.0547

De Miguel, A., De Miguel, M.D., Lucena-Antón, D., \& Rubio, M.D. (2018). Efectos de la Hipoterapia sobre la función motora en personas con síndrome de Down: revisión sistemática. Revista de Neurología, 67, 233-41. https: //dx.doi.org/10.33588/ rn.6707.2018117

Del Rosario-Montejo, O., Molina-Rueda, F., MuñozLasa, S., \& Alguacil-Diego, I.M. (2014). Efectividad de la terapia ecuestre en niños con retraso psicomotor. Neurología, 30(7), 425-432. https: // doi.org/10.1016/j.nrl.2013.12.023

Eizaguirre, B., Alonso, R., López, P.A., \& Díaz-Videla, M. (2020). Intervenciones asistidas con animales en pacientes con esclerosis múltiple. Calidad de vida y Salud, 13, 175-183.

Eraud, A. (2020). Una propuesta de intervención de equinoterapia para niños con TDAH desde una perspectiva de protección animal. Derecho Animal (Forum of Animal Law Studies), 11(2), 107-125. https:/ / doi.org/10.5565/rev/da.495

Fernández-Gutiérrez, C., Apolo-Arenas, M.D., Martínez-García, Y., \& Caña-Pino, A. (2014). Efectos de la Hipoterapia en la estabilidad postural en parálisis cerebral infantil: a propósito de un caso clínico. Fisioterapia, 37(3), 135-139. https: / / doi.org/ 10.1016/j.ft.2014.10.002

Fierro-Aldana, C.A., Tula-Garzón, E., \& Vera-Cárdenas, V.J. (2013). Efecto de la hipoterapia en áreas cognitivas y psicomotoras en pacientes con retraso psicomotor. Revista Facultad de Salud, 5(2), 70-78.

Fierro-Bósquez, M-J., Fierro-Vasco, S., \& Aguinaga, L.B. (2018). La equinoterapia en niños con trastornos del espectro autista. Revista Científica Mundo de la Investigación y el Conocimiento, 2(3), 650-665. https:// doi.org/10.26820/recimundo/2.(3).julio.2018.650665

Fine, A.H. (2010). Animals and therapists: Incorporating animals into psychotherapy: Guidelines and suggestions for therapists. Fine, A., editor. Handbook on animalassisted therapy: Theoretical foundations and guidelines for practice. 3. San Diego: Academic 


\section{Press.}

Gámez-Calvo, L., Gamonales, J.M., Silva-Ortiz, A.M., \& Muñoz-Jiménez, J. (2020). Benefits of hippotherapy in elderly people: Scoping review. Journal of Human Sport and Exercise, in press. https: / / doi.org/10.14198/jhse.2022.172.06

Gámez-Calvo, L., Silva-Ortiz, A., Gamonales, J.M., \& Muñoz-Jiménez, J. (2019). Influencia de la Hipoterapia en la calidad de vida de los niños con síndrome de Down: Revisión literaria. I Congreso Nacional Mujer y Deporte Paralímpico. Universidad de Huelva, Huelva (España).

Gamonales, J.M., Durán-Vaca, M., Gámez-Calvo, L., Hernández-Beltrán, V., \& Muñoz-Jiménez, J. (2021). Fútbol para personas con amputaciones: revisión sistemática exploratoria. RETOS: Nuevas Tendencias en Educación Física, Deportes y Recreación, 42(4), 145-153. https: / / doi.org/10.47197/retos.v42i0.86380

Gamonales, J.M., Gil-Sánchez, O., Porro-Cerrato, C., Gómez-Carmona, C.D., Mancha-Triguero, D., \& Gamonales, F.J. (2018). Psicomotricidad en el aula de Educación Infantil: alumnos con Trastorno de Déficit de Atención e Hiperactividad. Revista Profesional de Investigación, Docencia y Recursos Didácticos, 100(11), 440-454.

Gamonales, J.M., Muñoz-Jiménez, J., León-Guzmán, K., \& Ibáñez, S.J. (2018). Football 5-a-side for individuals with visual impairments: a review of the literature. European Journal of Adapted Physical Activity, 11(1), 1-19. https: / /doi.org/10.5507/euj.2018.004

García-Gómez, A., Rodríguez-Jiménez, M., Guerrero-Barona, E., Rubio-Jiménez, J. C., García-Peña, I., \& Moreno-Manso, J. M. (2016). Benefits of an experimental program of equestrian therapy for children with ADHD. Research in Developmental Disabilities, 59, 176-185. https://doi.org/10.1016/ j.ridd.2016.09.003

Gilboa, Y., \& Helmer, A. (2020). Self-Management Intervention for Attention and Executive Functions Using Equine-Assisted OccupationalTherapy among Children Aged 6-14 Diagnosed with Attention Deficit/Hyperactivity Disorder. Journal of Alternative and Complementary Medicine, 26(3), 239-246. https: / /doi.org/10.1089/acm.2019.0374

Hyun, G.J., Jung, T.W., Park, J.H., Kang, K.D., Kim, S.M., Son, Y.D., ... Han, D.H. (2016). Changes in Gait Balance and Brain Connectivity in Response to Equine-Assisted Activity and Training in Children with Attention Deficit Hyperactivity Disorder. Journal of Alternative and Complementary Medicine, 22(4), 286-293.https: / / doi.org/10.1089/acm.2015.0299
Jang, B., Song, J., Kim, J., Kim, S., Lee, J., Shin, H.Y., ... Joung, Y. S. (2015). Equine-Assisted Activities and Therapy for Treating Children with AttentionDeficit/Hyperactivity Disorder. Journal of Alternative and Complementary Medicine, 21(9), 546-553. https:/ /doi.org/10.1089/acm.2015.0067

Jiménez, A. (2017). Efectos de las terapias ecuestres en personas con parálisis cerebral. Revista Española de Discapacidad, 5(2), 171-184. https: / / doi.org/ 10.5569/2340-5104.05.02.09

Law, M., Stewart, D., Letts, L., Pollock, N., Bosch, J., \& Westmoreland, M. (1998). Guidelines for critical review of qualitative studies. McMaster University Occupational Therapy Evidence-based Practice Research Group, 1-9.

Lee, N., Park, S., \& Kim, J. (2015). Effects of hippotherapy on brain function, BDNF level, and physical fitness in children with ADHD. Journal of Exercise Nutrition \& Biochemistry, 19(2), 115-121.https:/ /doi.org/10.5717 / jenb.2015.15061209

Lee, N., Park, S., \& Kim, J. (2017). Hippotherapy and neurofeedback training effect on the brain function and serum brain-derived neurotrophic factor level changes in children with attention-deficit or/and hyperactivity disorder. Journal of Exercise Nutrition \& Biochemistry, 21(3), 35-42. https://doi.org/ 10.20463/jenb.2017.0018

Lomas, A., \& Clemente, A.L. (2017). Beneficios de la actividad físico-deportiva en niños y niñas conTDAH. EmásF: Revista Digital de Educación Física 44(8), 63-78.

López-Roa, L.M. (2011). Efectos de la hipoterapia en posición sedente hacia adelante en un paciente con retraso psicomotor e hipotonía. Memorias, 9(16), 130137.

López-Roa, L.M., \& Moreno-Rodríguez, E.D. (2015). Hipoterapia como técnica de habilitación y rehabilitación. Universidad y Salud, 17(2), 271-279. http: / / dx.doi.org/10.22267/rus.151702.11

López-Serrano, S., Marques, N.E., Suarez-Manzano, S., \& De la Torre-Cruz, M. (2019). Análisis preliminar de las relaciones entre el nivel de condición física y el apoyo parental percibido para la práctica deportiva en adolescentes con sobrepeso y obesidad. RETOS: Nuevas Tendencias en Educación Física, Deportes y Recreación, 37(1), 527-531. https://doi.org/10.47197/ retos.v37i37.71599

Mallon, G.P., Ross, S.B., Ross, L., \& Klee, S. (2010). Designing and implementing animal-assisted therapy programs in health and mental health organizations: Rules and principles to guide program development. In: Fine, A., editor. Handbook on animal-assisted therapy: 
Theoretical foundations and guidelines for practice. San Diego: Academic Press.

Mendizábal-Alonso, P. (2020). Intervenciones fisioterápicas mediante hipoterapia en el tratamiento de la parálisis cerebral infantil. Revisión bibliográfica. Rehabilitación, 54(2), 96-106. https://doi.org/ 10.1016/j.rh.2019.11.003

Montero, I., \& León, O.G. (2007). A guide for naming research studies in Psychology. International Journal of Clinical and Health Psychology, 7(3), 847-862.

Muñoz-Lasa, S., López, C., Atín-Arratibel, M.Á., Bravo-Llatas, C., Pastor-Jimeno, S., \& Máximo-Bocanegra, N. (2019). Efecto de la hipoterapia en esclerosis múltiple: estudio piloto en calidad de vida, espasticidad, marcha, suelo pélvico, depresión y fatiga. Medicina Clínica, 152(2), 55-58.

Oh,Y., Joung,Y.S., Jang, B., Yoo, J.H., Song, J., Kim, J., ... Jeong, B. (2018). Efficacy of Hippotherapy Versus Pharmacotherapy in Attention-Deficit/ Hyperactivity Disorder: A Randomized ClinicalTrial. Journal of Alternative and Complementary Medicine, 24(5), 463-471.https://doi.org/10.1089/acm.2017.0358

Orjales, I., \& Polaino-Lorente, A. (2014). Programas de intervención cognitivo-conductual para niños con déficit de atención con hiperactividad. Cepe. Ciencias Educación Preescolar Especial. ISBN 10: 8478693599 / ISBN 13: 9788478693597

Ortega-Espinoza, S.I. (2021). Método Kawuallu como estrategia en el desarrollo psicomotriz de niños con trastorno del espectro autista (Bachelor's thesis). Universidad del Azuay, Cuenca (Ecuador).

Pérez-Rodríguez, C. (2012). Las revisiones sistemáticas: declaración PRISMA. Revistas Española de Nutrición Comunitaria, 18(1), 57-58.

Pérez Gómez, J., Amigo Gamero, H., Collado Mateo, D., Barrios Fernández, S., Muñoz Bermejo, L., García Gordillo, M.Á., Carlos-Vivas, J., \& Adsuar, J.C. (2020). Equine assisted activities and therapies in children with attention deficit/hyperactivity disorder: A systematic review. Journal of Psychiatric and Mental Health Nursing. https: / / doi.org/10.1111/ jpm. 12710

Rivera-Ramo, F. (2020). Revisión de la terapia ocupacional y la intervención asistida con animales en personas con trastorno del espectro autista (Trabajo de fin de grado). Universidad da Coruña, Coruña (España).

Sarmento, H., Clement, F.M., Araújo, D., Davids, K., McRobert, A., \& Figueiredo, A. (2018). What performance analysts need to know about research trends in Association Football (2012-2016): a systematic review. Sports Medicine, 48, 799-836. https: / doi.org/10.1007/s40279-017-0836-6

Shurtleff, T., \& Engsberg, J. (2012). Long-term effects of hippotherapy on one child with cerebral palsy: A research case study. British Journal of Occupational Therapy, 75(8), 359-366. https: / /doi.org/10.4276/ $030802212 X 13433105374279$

Silva-Ortiz, A.M., Gamonales, J.M., Gámez-Calvo, L., \& Muñoz-Jiménez, J. (2020). Benefits of inclusive physical activity for people with Down Syndrome: systematic review. Revista Euroamericana de Ciencias Del Deporte, 9(2), 81-94.

Tabares, C., Vicente-Castro, F., Sánchez-Herrera, S., Alejo, S., \& Cubero, J. (2014). Cambios hormonales por efecto de la terapia asistida con caballos en personas autistas. Campo Abierto. Revista de Educación, 33(1), 37-46.

Thomas, J.R., Silverman, S.J., \& Nelson, J.K. (2015). Research Methods in Physical Activity ( $\left.7^{\mathrm{a}} \mathrm{Ed}\right)$. Campaing: Human Kinetics.

Uribe,A.M., Restrepo, T.F., \&Yajaira, D. (2012). ¿Cómo beneficia la Equinoterapia a las personas con síndrome de Down? Revista CES Salud Pública, 3(1), 4-10.

Vargas, J., Patricio, L., Solís-Cartas, U., MartínezLarrarte, J.P., \& Serrano-Espinosa, I. (2016). Aplicación de la Hipoterapia en los niños con parálisis cerebral. Revista Archivo Médico de Camagüey, 20(5), 496-506.

Villasís-Keever, M.Á., \& Pineda-Leguízamo, R. (2017). Utilidad de hipoterapia en la parálisis cerebral infantil. Revista Mexicana de Pediatría, 84(4), 131-133.

White, E., Zippel, J., \& Kumar, S. (2020). The effect of equine-assisted therapies on behavioural, psychological and physical symptoms for children with attention deficit/hyperactivity disorder: A systematic review. Complementary Therapies in Clinical Practice, 39(September 2019), 101101. https:// doi.org/10.1016/j.ctcp.2020.101101

Wigal, S.B., Emmerson, N., Gehricke, J. \& Galassetti, P. (2013). Exercise: Applications to childhood ADHD. Journal of Attention Disorders, 17 (4), 279-290.

Yoo, J.H., Oh, Y., Jang, B., Song, J., Kim, J., Kim, S., ... Joung,Y.S. (2016). The effects of equine-assisted activities and therapy on resting-state brain function in attention-deficit/hyperactivity disorder: A pilot study. Clinical Psychopharmacology and Neuroscience, 14(4), 357-364. https://doi.org/10.9758/ cpn.2016.14.4.357

Ziereis, S., \& Jansen, P. (2015). Effects of physical activity on executive function and motor performance in children with ADHD. Research in Developmental Disabilities, 38 , 181-191. 